\title{
Narcotic Consumption Following Minimally Invasive Lumbar Decompression: A Comparison Between Hospital and Ambulatory-Based Surgery Centers
}

\author{
BENJAMIN KHECHEN, BA, BRITTANY E. HAWS, MD, MUNDEEP S. BAWA, BA, DIL V. PATEL, BS, \\ HARMEET S. BAWA, BA, DUSTIN H. MASSEL, MD, BENJAMIN C. MAYO, MD, KAITLYN L. CARDINAL, \\ BA, JORDAN A. GUNTIN, BS, KERN SINGH, MD \\ Department of Orthopaedic Surgery, Rush University Medical Center, Chicago, Illinois
}

\begin{abstract}
Background: Several studies have compared outcomes between hospital-based centers (HBCs) and ambulatory surgery centers (ASCs) following minimally invasive lumbar decompression (MIS LD). However, the association between narcotic consumption and pain in the immediate postoperative period has not been well characterized. As such, this study aims to examine pain, narcotic consumption, and length of stay (LOS) among patients discharged on postoperative day 0 following a 1-level MIS LD between HBCs or ASCs.

Methods: Patients who underwent a primary, 1-level MIS LD were retrospectively reviewed and stratified by operative location. Differences between groups in patient demographics were assessed using independent-sample $t$ tests for continuous variables and $\chi^{2}$ analysis for categoric variables. The operative location and its effect on perioperative characteristics, inpatient pain scores, and narcotics consumption were analyzed using multivariate linear regression adjusted for significant patient characteristics.

Results: There were 235 patients identified, of whom 90 and 145 underwent surgery at an $\mathrm{HBC}$ or ASC, respectively. The $\mathrm{HBC}$ cohort exhibited an increased comorbidity burden and had a greater percentage of privately insured patients. The HBC cohort recorded shorter operative time and greater total estimated blood loss. Patients in the HBC cohort experienced prolonged LOS, and consumed greater total oral morphine equivalents compared with the ASC cohort. No differences were observed in the remaining outcomes.

Conclusions: The results of the current study suggest that patients who underwent MIS LD at an ASC received fewer narcotics than patients treated at an HBC, which may contribute to shortened LOS. Additionally, there was no difference in patient-reported pain between cohorts despite the differences in narcotic use. As such, postoperative narcotics administration varied, indicating HBC patients perhaps required more narcotic pain medications to achieve the same pain scores that were sufficient enough to allow patient discharge, thus prolonging LOS.
\end{abstract}

Level of Evidence: III

Minimally Invasive Surgery

Keywords: lumbar decompression, narcotic consumption, inpatient pain, same-day discharge, hospital, ambulatory surgery center

\section{INTRODUCTION}

Minimally invasive lumbar decompression (MIS LD) is one of the most common spinal procedures performed for the treatment of degenerative conditions. ${ }^{1,2}$ As the US population ages, the need for surgical treatment of degenerative conditions in elderly patients will continue to increase. ${ }^{1,3,4}$ With the rising demand for treatment of degenerative spinal pathology, outpatient surgery has received the attention of hospital institutions and private practices alike as a mechanism for efficient delivery of care. ${ }^{4-6}$ Several studies have demonstrated the feasibility, efficacy, and safety of outpatient lumbar decompression. $^{3-8}$ When compared to hospitalbased procedures, outpatient lumbar decompression procedures have demonstrated shorter operative and recovery times, reduced hospital costs, improved patient satisfaction, and risk of perioperative complications. ${ }^{2,4,9-11}$ As such, outpatient lumbar decompression has been increasingly used.

Narcotic consumption remains a significant concern within the spine community. The prevalence of narcotic use in the treatment of acute and chronic pain leads to the potential for dependence and abuse. ${ }^{12-14}$ As such, minimizing preoperative 
and postoperative narcotic use following MIS LD is an important topic of investigation. Despite evidence demonstrating successful clinical outcomes following outpatient MIS LD, few studies have evaluated narcotic use in the immediate postoperative period. In this context, the aim of the current study is to evaluate early postoperative pain and narcotic consumption between MIS LD performed in a hospital versus ambulatory setting.

\section{MATERIALS AND METHODS}

\section{Patient Population}

Institutional review board approval was obtained for this study (ORA no. 14051301). A prospectively maintained surgical registry of patients was retrospectively reviewed. Patients were included in this analysis if they underwent a primary, single-level MIS LD for degenerative lumbar spine pathology between 2013 and 2017. Each MIS LD procedure included a foraminotomy, facetectomy, laminectomy, and discectomy. All procedures were performed by the senior author (K.S.) at a single institution. Patients were stratified by operative location: a hospital-based center (HBC) or an ambulatory surgery center (ASC). Only patients who were discharged on the day of surgery were included in the analysis. Pain management was standardized according to a multimodal analgesia protocol developed by a team of surgeons and anesthesiologists at our institution.

\section{Data Collection}

All data were obtained from the aforementioned surgical registry. Patients were classified according to demographic, comorbidity, and operative factors. Demographic factors included age, sex, smoking status, body mass index $\left(<30 \mathrm{~kg} / \mathrm{m}^{2}, \geq 30 \mathrm{~kg} / \mathrm{m}^{2}\right)$, and primary insurance payer. Comorbidity was analyzed with the Charlson Comorbidity Index. Perioperative factors, such as operative time, estimated intraoperative blood loss, and length of stay, were recorded. Inpatient visual analog scale pain scores, as assessed by the nursing staff according to normal protocols, were averaged over the duration of stay. Inpatient narcotic consumption was recorded in oral morphine equivalents (OMEs), using the conversion ratios described by Gordon et al ${ }^{15}$ Narcotic consumption was evaluated as a sum of the total OMEs consumed on postoperative day 0 and as an average over the duration of stay.

Perioperative complications were also recorded, including transient urinary retention, altered mental status, ileus, postoperative transfusion requirement, cardiac arrhythmia, acute renal failure, urinary tract infection, aspiration/reintubation, deep venous thrombosis, pulmonary embolism, pneumothorax, and pneumonia. Transient urinary retention was classified as a complication if postoperative recatheterization was required.

\section{Statistical Analysis}

Statistical analysis was performed using Stata/ MP 13.1 for Mac (StataCorp LP, College Station, Texas). Differences between groups in patient demographics were assessed using independentsample $t$ tests for continuous variables and $\chi^{2}$ analysis for categoric variables. The operative location and its effect on perioperative characteristics, inpatient pain scores, and narcotics consumption were analyzed using multivariate linear regression adjusted for significant patient characteristics. A $P$ value $<.05$ was used to determine statistical significance.

\section{RESULTS}

A total of 235 patients who underwent a primary 1-level MIS LD were included in the analysis. Of these, $90(38.3 \%)$ underwent surgery at an HBC and $145(61.7 \%)$ at an ASC. The average patient age was 41.4 years for the HBC cohort and 40.7 years for the ASC cohort $(P=.632)$. Most of the patients in both cohorts were male (HBC, 67.7\%; ASC, 75.9\%; $P=$ .126). A greater number of patients in the $\mathrm{HBC}$ cohort held private insurance compared with patients in the ASC cohort $(76.7 \%$ vs. $37.2 \%, P=$ .026). Patients in the HBC cohort exhibited a higher Charlson Comorbidity Index compared with the ASC cohort (1.1 vs. $0.8, P=.041)$. The HBC patients also reported no difference in preoperative visual analog scale back pain scores compared with the ASC cohort (6.6 vs. $6.0, P=.144$ ). Similarly, no difference was determined between the HBC and ASC cohorts when comparing preoperative visual analog scale leg pain scores (6.5 vs. $6.2, P=.938)$. No other significant differences in preoperative demographics existed between cohorts (Table 1).

Table 2 details the perioperative outcomes between groups. Patients in the HBC cohort 
Table 1. Baseline characteristics. ${ }^{a}$

\begin{tabular}{|c|c|c|c|}
\hline & Hospital-Based Center $(n=90)$ & Ambulatory Surgery Center $(n=145)$ & $P$ Value ${ }^{b}$ \\
\hline Age, $y$, mean $\pm S D$ & $41.4 \pm 12.4$ & $40.7 \pm 11.78$ & .632 \\
\hline Sex, \% (n) & & & .126 \\
\hline Female & $33.3(30)$ & $24.1(35)$ & \\
\hline Male & $67.7(60)$ & 75.9 (110) & \\
\hline Smoking status, \% (n) & & & .290 \\
\hline Nonsmoker & $82.2(74)$ & $76.4(110)$ & \\
\hline Smoker & $17.8(16)$ & $22.6(34)$ & \\
\hline BMI, \% (n) & & & .138 \\
\hline$<30 \mathrm{~kg} / \mathrm{m}^{2}$ & $60.0(54)$ & $69.4(100)$ & \\
\hline$\geq 30 \mathrm{~kg} / \mathrm{m}^{2}$ & $40.0(36)$ & $30.6(44)$ & \\
\hline Insurance, \% (n) & & & .026 \\
\hline Worker's compensation & $23.3(21)$ & $62.8(91)$ & \\
\hline Private insurance & $76.7(69)$ & $37.2(54)$ & \\
\hline Comorbidity burden (CCI) & $1.1 \pm 1.5$ & $0.8 \pm 1.0$ & .041 \\
\hline Preoperative VAS back pain, mean \pm SD & $6.3 \pm 2.2$ & $5.9 \pm 2.6$ & .144 \\
\hline Preoperative VAS leg pain, mean \pm SD & $6.5 \pm 2.3$ & $6.2 \pm 2.5$ & .938 \\
\hline
\end{tabular}

Abbreviations: BMI, body mass index; CCI, Charlson Comorbidity Index; VAS, visual analog scale.

${ }^{a}$ Boldface indicates statistical significance.

${ }^{\mathrm{b}} P$ value was calculated for each category using $\chi^{2}$ analysis (categoric), Student $t$ test (continuous), and multivariate linear regression controlling for insurance status and CCI.

demonstrated shorter operative times (30.9 vs. 41.3 minutes, $P<.001)$ and increased total estimated blood loss (34.4 vs. $26.3 \mathrm{~mL}, P<.001)$ compared with the ASC cohort. However, these differences were not clinically significant. The HBC cohort exhibited longer lengths of stay (5.8 vs. 2.6 hours, $P$ $<.001)$ than the ASC cohort. When examining pain scores, there were no differences observed between cohorts (HBC, 3.7 versus ASC, 3.9; $P=.714$ ). Two patients who underwent MIS LD at an $\mathrm{HBC}$ experienced transient urinary retention that required recatheterization. $P$ values were calculated using multilinear regression controlling for insurance status and Charlson Comorbidity Index. No other complications were identified in either group.

Table 3 describes the OME consumption on postoperative day 0 between cohorts. Patients in the HBC cohort consumed greater total OMEs (35.7 vs. 15.0, $P<.001)$ compared with the ASC cohort (Figure 1). The HBC cohort consumed greater amounts of hydrocodone (13.8 vs. 4.2 OMEs, $P<$ $.001)$ and fentanyl (16.7 vs. 2.3 OMEs, $P<.001$ ). The ASC cohort consumed greater amounts of tramadol (5.0 vs. 3.3 OMEs, $P=.028)$ and oxycodone (3.8 vs. 1.7, $P=.053$ ); however, this did not reach statistical significance. $P$ values were calculated using multilinear regression controlling for insurance status and Charlson Comorbidity Index. Figure 2 illustrates the percentage of medications contributing to total OME administered in each setting. Hydrocodone and fentanyl were the most used narcotics in the HBC $(46.2 \%$ and $28.7 \%$ ). In contrast, tramadol was the most commonly administered narcotic in the ASC $(39.6 \%)$, followed by hydrocodone $(30.8 \%)$.

\section{DISCUSSION}

With the current trend toward outpatient spinal procedures, recent literature has emphasized the setting's safety and efficacy compared with hospitalbased procedures. ${ }^{1-8,11,14,16}$ However, there has been limited research comparing narcotic administration between these two settings, specifically in the immediate postoperative period. Narcotic consumption is associated with a significant side effect profile, including drowsiness, nausea, vomiting,

Table 2. Perioperative outcomes. ${ }^{a}$

\begin{tabular}{lccc}
\hline & Hospital-Based Center $(\mathbf{n}=\mathbf{9 0})$ & Ambulatory Surgery Center $(\mathbf{n}=\mathbf{1 4 5}) \boldsymbol{P}$ Value \\
\hline Operative time, min, mean \pm SD & $\mathbf{3 0 . 9} \pm \mathbf{1 2 . 6}$ & $\mathbf{4 1 . 3} \pm \mathbf{1 5 . 8}$ & $<.001$ \\
Estimated blood loss, mL, mean \pm SD & $\mathbf{3 4 . 4} \pm \mathbf{1 2 . 3}$ & $\mathbf{2 6 . 3} \pm \mathbf{7 . 9}$ & $\mathbf{2 . 0 0 1}$ \\
Length of hospital stay, hr, mean \pm SD & $\mathbf{5 . 8} \pm \mathbf{2 . 2}$ & $\mathbf{2 . 4}$ & $3.9 \pm 2.4$ \\
Inpatient VAS pain scores POD 0, mean \pm SD & $3.7 \pm 1.9$ & $0.0 \%(0)$ & .714 \\
Perioperative complications, \% (n) & $2.2 \%(2)$ & - \\
\hline
\end{tabular}

Abbreviations: POD, postoperative day; VAS, visual analog scale.

${ }^{a}$ Boldface indicates statistical significance.

${ }^{\mathrm{b}} P$ values calculated using multivariate linear regression controlling for insurance status and Charlson Comorbidity Index.

${ }^{c}$ Perioperative complications included transient urinary retention requiring recatheterization $(\mathrm{n}=2)$. 
Table 3. Oral morphine equivalent consumption on postoperative day $0 .^{a}$

\begin{tabular}{|c|c|c|c|}
\hline & Hospital-Based Center $(\mathrm{n}=90)$ & Ambulatory Surgical Center $(n=145)$ & $P$ Value $^{\mathrm{b}}$ \\
\hline Total OME Consumption POD 0, mean \pm SD & $35.7 \pm 25.3$ & $15.0 \pm 14.3$ & $<.001$ \\
\hline Hourly OME consumption POD 0 , mean \pm SD & $6.9 \pm 5.3$ & $6.4 \pm 6.4$ & .325 \\
\hline \multicolumn{4}{|l|}{$\mathrm{OME}$ dose, mean $\pm \mathrm{SD}$} \\
\hline Hydrocodone & $13.8 \pm 9.8$ & $4.2 \pm 7.0$ & $<.001$ \\
\hline Fentanyl & $16.7 \pm 23.5$ & $2.3 \pm 7.5$ & $<.001$ \\
\hline Tramadol & $3.3 \pm 5.2$ & $5.0 \pm 6.1$ & .028 \\
\hline Oxycodone & $1.8 \pm 5.2$ & $3.5 \pm 7.7$ & .053 \\
\hline Morphine & $0.2 \pm 1.6$ & $0.1 \pm 0.7$ & .499 \\
\hline
\end{tabular}

Abbreviations: OME, oral morphine equivalent; POD, postoperative day.

${ }^{a}$ Boldface indicates statistical significance.

${ }^{\mathrm{b}} P$ values calculated using multivariate linear regression controlling for insurance status and Charlson Comorbidity Index.

constipation, difficulty with deep breathing, coughing, impaired bowel and bladder function, and transient impairment of psychomotor skills. ${ }^{17-19}$ The potential hazards of narcotic toxicity have resulted in an effort to reduce the amount of postoperative narcotic consumption. In this context, the goal of the current study was to measure narcotic consumption among patients who underwent MIS LD in an ASC versus HBC.

The results of the present study suggest patients who underwent MIS LD at an ASC consumed significantly less total narcotics compared with patients who underwent MIS LD at an HBC. Additionally, patients in both cohorts reported similar pain levels prior to discharge despite patients in the ASC cohort receiving less total narcotic medication. When comparing total OME consumption, more than two thirds $(73.8 \%)$ of the ASC cohort consumed less than or equal to $20 \mathrm{mg}$ OME. In contrast, only one third $(32.2 \%)$ of the HBC cohort consumed less than $20 \mathrm{mg}$ OME. Relative to total OME consumption, the HBC administered a

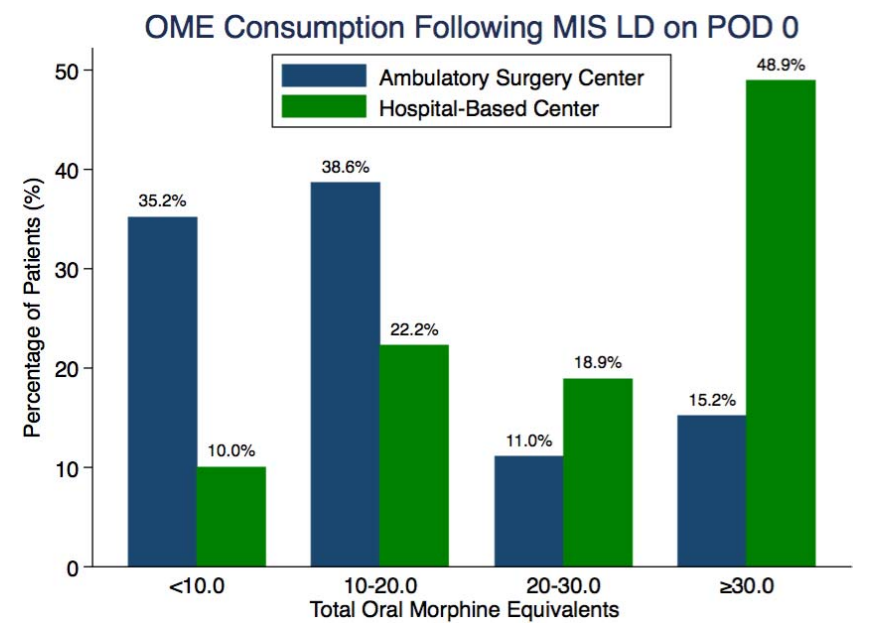

Figure 1. Bar graph illustrating the total oral morphine equivalent consumption between cohorts. Abbreviations: OME, oral morphine equivalents; MIS LD, minimally invasive lumbar decompression; POD, postoperative day. greater percentage of hydrocodone and fentanyl, whereas the ASC administered a larger percentage of the less-potent narcotic, tramadol. The ASC patients received a reduced quantity of high-potency narcotics, instead receiving an increased quantity of a lower-potency narcotic, which may have contributed to their decreased length of stay. The reduced narcotic administration in the ASC may be reflective of a greater motivation to discharge patients in this setting in a timely manner. Models of care designed used in same-day surgery populations have sought to reduce narcotic administration as a means to promote cost-efficiency and to expedite discharge. $^{20-23}$

Excess narcotic administration in the early postoperative period has been associated with a significant side effect profile and can contribute to delayed discharge. ${ }^{17-19,24,25}$ In a retrospective analysis of a heterogenous orthopedic population, Hansen et $\mathrm{al}^{26}$ investigated the effect of narcotic consumption on length of stay between patients receiving a combination of intravenous (IV) acetaminophen and IV narcotics to patients receiving IV

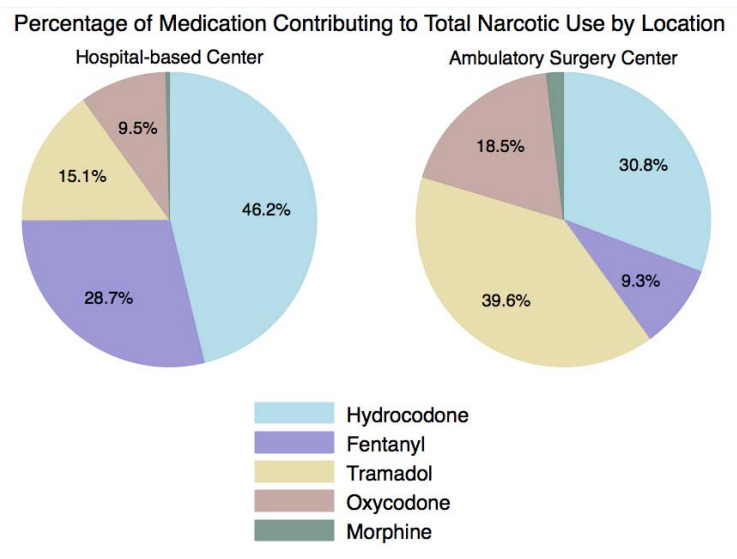

Figure 2. Pie chart illustrating the percentage of medication contributing to total narcotic administration in each setting. 
narcotics alone. The authors demonstrated patients receiving a combination of narcotic and nonnarcotic medication recorded shortened lengths of stay compared with patients receiving narcotic medication alone. In a prospective randomized study, Padda et $\mathrm{al}^{27}$ investigated the recovery profile of ophthalmologic surgical patients comparing the use of rectal acetaminophen or intravenous fentanyldroperidol. Study results illustrated that patients receiving fentanyl experienced longer time in the postsurgical unit compared with the acetaminophen-treated group. The authors concluded the administration of fentanyl provided no discrete benefit over acetaminophen.

Although patients at the ASC received less narcotics than the HBC patients, there were no differences in pain scores observed between cohorts. These results suggest patients undergoing MIS LD can receive a reduced quantity of narcotics in the immediate postoperative period without conceding adequate pain control. Previous studies investigating the efficacy of narcotic alternatives, such as tramadol, for treatment of postoperative pain have demonstrated that the centrally acting analgesic provides less sedation, less euphoria, and less respiratory depression compared with frequently administered narcotic agents. ${ }^{28-30}$ In a randomized controlled trial by $\mathrm{Ng}$ et $\mathrm{al}^{31}{ }^{31}$ patient-controlled analgesia administration of tramadol and parenteral fentanyl were investigated to determine effect on pain relief following abdominal surgery. When comparing verbal pain scores, no differences were found between groups at rest in the first 24 hours (fentanyl group, 4.2; tramadol group, 3.6; $P=.417)$. The authors demonstrated the use of a narcotic alternative, such as tramadol, can produce equivalent analgesia, without the use of the higher-potency narcotic, fentanyl. In the current study, no differences in pain scores were observed between cohorts despite the differences in fentanyl administration between groups. However, it is imperative to also consider that patients in the $\mathrm{HBC}$ setting could potentially have needed more narcotics in order to have equal pain relief relative to the patients in the ASC setting. As such, that patients in each setting achieved the same reported pain outcomes indicates hospital-based patients perhaps require more pain medication than those at an ASC. Further study is required in order to reassess postoperative narcotic medication administrative schedules in order to reduce high-potency narcotic medication and increased use of narcotics alternatives and lower-potency narcotics.

The current study is not without limitations. First, all procedures were performed by a single surgeon, limiting the generalizability to the greater population Second, because of the retrospective nature of the study, there may have been a selection bias between cohorts, because patients at greater preoperative risk for adverse events are more likely to be treated at an HBC than at an ASC for possible hospital admission. Third, a potential for a confounding variable may exist to explain shorter operative times and prolonged length of stay in the patients treated at the HBC. However, only 2 postoperative adverse events were recorded, and both occurred in the HBC cohort. Additionally, the risk of bias was minimized by use of statistical analyses that adjusted for the preoperative characteristics that varied between cohorts. Lastly, no measurement of preoperative narcotic use was available, resulting in the inability to quantify the effect of narcotic tolerance on postoperative pain scores and narcotics use.

\section{CONCLUSIONS}

To further advance the safety and efficacy of outpatient lumbar decompression procedures, the investigation of differences in narcotic consumption in the early postoperative period is necessary. The results of the current study suggest that patients who underwent MIS LD at an ASC receive fewer narcotics than patients treated at an $\mathrm{HBC}$, which may contribute to shortened length of stay. Additionally, there was no difference in patient-reported pain between cohorts despite the differences in narcotic administration. As such, postoperative narcotics administration varied, indicating $\mathrm{HBC}$ patients required more narcotic pain medications to achieve the same pain scores that were sufficient to allow patient discharge, thus prolonging length of stay.

\section{ACKNOWLEDGMENTS}

Preliminary portions of this work have been presented at the Society for Minimally Invasive Spine Surgery in 2016 and 2017 and at The International Society for the Advancement of Spine Surgery in 2017. 


\section{REFERENCES}

1. Gray DT, Deyo RA, Kreuter W, et al. Population-based trends in volumes and rates of ambulatory lumbar spine surgery. Spine (Phila Pa 1976). 2006;31(17):1957-1963; discussion 1964.

2. Pugely AJ, Martin CT, Gao Y, Mendoza-Lattes SA. Outpatient surgery reduces short-term complications in lumbar discectomy: an analysis of 4310 patients from the ACS-NSQIP database. Spine (Phila Pa 1976). 2013;38(3):264-271.

3. Best NM, Sasso RC. Success and safety in outpatient microlumbar discectomy. J Spinal Disord Tech. 2006;19(5):334337.

4. Singhal A, Bernstein M. Outpatient lumbar microdiscectomy: a prospective study in 122 patients. Can J Neurol Sci. 2002;29(3):249-252.

5. Techy F, Benzel EC. Implementing an outpatient ambulatory discectomy protocol at a large academic center: a change for the better. World Neurosurg. 2015;83(3):341-342.

6. Lang SS, Chen HI, Koch MJ, et al. Development of an outpatient protocol for lumbar discectomy: our institutional experience. World Neurosurg. 2014;82(5):897-901.

7. Best NM, Sasso RC. Outpatient lumbar spine decompression in 233 patients 65 years of age or older. Spine (Phila $P a$ 1976). 2007;32(10):1135-1139; discussion 1140.

8. Zahrawi F. Microlumbar discectomy: is it safe as an outpatient procedure? Spine (Phila Pa 1976). 1994;19(9):10701074.

9. An HS, Simpson JM, Stein R. Outpatient laminotomy and discectomy. J Spinal Disord. 1999;12(3):192-196.

10. Asch HL, Lewis PJ, Moreland DB, et al. Prospective multiple outcomes study of outpatient lumbar microdiscectomy: should 75 to $80 \%$ success rates be the norm? J Neurosurg. 2002;96(1 suppl):34-44.

11. Bookwalter JW 3rd, Busch MD, Nicely D. Ambulatory surgery is safe and effective in radicular disc disease. Spine (Phila Pa 1976). 1994;19(5):526-530.

12. Hyer LA, Walid MS, Brooks AM, Darmohray DM, Robinson JS Jr. Interaction of age and opioid dependence on length of hospital stay for spine surgery patients. Psychol Rep. 2009;105(2):361-364.

13. Rodgers J, Cunningham K, Fitzgerald K, Finnerty E. Opioid consumption following outpatient upper extremity surgery. J Hand Surg Am. 2012;37(4):645-650.

14. Walid MS, Robinson JS 3rd, Robinson ER, Brannick BB, Ajjan M, Robinson JS Jr. Comparison of outpatient and inpatient spine surgery patients with regards to obesity, comorbidities and readmission for infection. J Clin Neurosci. 2010;17(12):1497-1498.

15. Gordon DB, Stevenson KK, Griffie J, Muchka S, Rapp C, Ford-Roberts K. Opioid equianalgesic calculations. J Palliat Med. 1999;2(2):209-218.

16. Helseth O, Lied B, Halvorsen CM, Ekseth K, Helseth E. Outpatient cervical and lumbar spine surgery is feasible and safe: a consecutive single center series of 1449 patients. Neurosurgery. 2015;76(6):728-737; discussion 737-728.

17. Rajpal S, Gordon DB, Pellino TA, et al. Comparison of perioperative oral multimodal analgesia versus IV PCA for spine surgery. J Spinal Disord Tech. 2010;23(2):139-145.

18. Kehlet H, Dahl JB. Anaesthesia, surgery, and challenges in postoperative recovery. Lancet. 2003;362(9399):1921-1928.
19. Mathiesen O, Dahl B, Thomsen BA, et al. A comprehensive multimodal pain treatment reduces opioid consumption after multilevel spine surgery. Eur Spine J. 2013;22(9):2089-2096.

20. White PF, Rawal S, Nguyen J, Watkins A. PACU fasttracking: an alternative to "bypassing" the PACU for facilitating the recovery process after ambulatory surgery. $J$ Perianesth Nurs. 2003;18(4):247-253.

21. Duncan PG, Shandro J, Bachand R, Ainsworth L. A pilot study of recovery room bypass ("fast-track protocol") in a community hospital. Can J Anaesth. 2001;48(7):630-636.

22. Williams BA, Kentor ML, Williams JP, et al. PACU bypass after outpatient knee surgery is associated with fewer unplanned hospital admissions but more phase II nursing interventions. Anesthesiology. 2002;97(4):981-988.

23. Apfelbaum JL, Walawander CA, Grasela TH, et al. Eliminating intensive postoperative care in same-day surgery patients using short-acting anesthetics. Anesthesiology. 2002;97(1):66-74.

24. Pizzi LT, Toner R, Foley K, et al. Relationship between potential opioid-related adverse effects and hospital length of stay in patients receiving opioids after orthopedic surgery. Pharmacotherapy. 2012;32(6):502-514.

25. Oderda GM, Said Q, Evans RS, et al. Opioid-related adverse drug events in surgical hospitalizations: impact on costs and length of stay. Ann Pharmacother. 2007;41(3):400-406.

26. Hansen RN, Pham A, Strassels SA, Balaban S, Wan GJ. Comparative analysis of length of stay and inpatient costs for orthopedic surgery patients treated with IV acetaminophen and IV opioids vs. IV opioids alone for post-operative pain. $A d v$ Ther. 2016;33(9):1635-1645.

27. Padda GS, Cruz OA, Krock JL. Comparison of postoperative emesis, recovery profile, and analgesia in pediatric strabismus repair: rectal acetaminophen versus intravenous fentanyl-droperidol. Ophthalmology. 1997;104(3):419-424.

28. Houmes RJ, Voets MA, Verkaaik A, Erdmann W, Lachmann B. Efficacy and safety of tramadol versus morphine for moderate and severe postoperative pain with special regard to respiratory depression. Anesth Analg. 1992;74(4):510-514.

29. Preston KL, Jasinski DR, Testa M. Abuse potential and pharmacological comparison of tramadol and morphine. Drug Alcohol Depend. 1991;27(1):7-17.

30. Sacerdote P, Bianchi M, Gaspani L, et al. The effects of tramadol and morphine on immune responses and pain after surgery in cancer patients. Anesth Analg. 2000;90(6):1411-1414.

31. Ng KF, Yuen TS, Ng VM. A comparison of postoperative cognitive function and pain relief with fentanyl or tramadol patient-controlled analgesia. J Clin Anesth. 2006;18(3):205-210.

Disclosures and COI: No funds were received in support of this work. No benefits in any form have been or will be received from any commercial party related directly or indirectly to the subject of this manuscript.

Corresponding Author: Kern Singh, MD, Department of Orthopaedic Surgery, Rush Univer- 
sity Medical Center, 1611 W Harrison St, Ste 300, Chicago, IL 60612. Phone: (312) 432-2373; Fax: (708) 492-5373; Email: kern.singh@rushortho.com.

Published 30 April 2019
This manuscript is generously published free of charge by ISASS, the International Society for the Advancement of Spine Surgery. Copyright (C) 2019 ISASS. To see more or order reprints or permissions, see http://ijssurgery.com. 